\title{
Análisis de objetos virtuales de aprendizaje centrados en el Principio de Le Chatelier
}

\author{
Virtual objects analysis base on the Principles of Le Chatelier
}

\section{*Edgar Ricardo Domínguez Prada \& *Henry Giovany Cabrera Castillo}

Domínguez, E.D., Cabrera, H. G. (2020). Análisis de objetos virtuales de aprendizaje centrados en el Principio de Le Chatelier. Convergencia Educativa, 7, julio, 1-21. DOI: http://doi.org/10.29035/rce.7.5

[Recibido: 30 marzo, 2020 / Aceptado: 18 abril, 2020]

\section{RESUMEN}

Este documento tiene el propósito de formular implicaciones a partir del análisis de OVA utilizados en la enseñanza del principio de Le Chatelier. El enfoque metodológico al que se adscribió este trabajo fue el cualitativo, de tipo descriptivo e interpretativo. Para el análisis se adaptaron cuatro instrumentos de evaluación enfocados en aspectos como: la población en la que se puede implementar el OVA, la pertinencia, relevancia e interactividad de cada material, se hizo la evaluación del número de ejemplos que cada OVA ofrece y si son suficientes para reforzar lo planteado en el contenido, de igual modo se evaluó la metodología empleada en cada material y la forma de motivar a los usuarios. La muestra estuvo conformada por cinco OVA gratuitos que estaban almacenados en diversos repositorios. Los resultados permitieron establecer que aún hace falta fortalecer aspectos como la interactividad, la metodología y el contenido de los OVA; muchos de los materiales analizados arrojaron resultados negativos en estos aspectos. A manera de conclusión, podemos decir que es necesario que los profesores en formación y profesores activos se hagan participes de los procesos de diseño y elaboración de OVA para, de este modo, se puedan corregir los errores presentados en los resultados de los materiales analizados.

Palabras clave: objetos virtuales de aprendizaje, TIC, material educativo computarizado, Principio de Le Chatelier, enseñanza de la química.

\footnotetext{
* Universidad del Bío Bío, Chillán, Chile.
} 
Domínguez Prada, E.D., Cabrera Castillo, H. G. (2020). Análisis de objetos virtuales de aprendizaje centrados en el Principio de Le Chatelier. Revista Convergencia Educativa, 7, julio, 1-21. DOI: http://doi.org/10.29035/rce.7.5

\begin{abstract}
This document aims to formulate implications from the analysis of VLO used in the teaching of the Le Chatelier's Principle. The methodological approach of this work was qualitative, of the kind of descriptive and interpretative. For the analysis, four evaluation instruments were adapted, focused on aspects such the following: the population in which the VLO can be implemented, the relevance, relevance and interactivity of each material. An evaluation was made of the number of examples that each VLO offers and whether they are enough to reinforce what is proposed in the content, as well was evaluated the methodology used in each material and the way to motivate users. The sample consisted of five free VLOs that were stored in different repositories. The results made possible to establish that there is still a need to strengthen aspects such as interactivity, methodology and the content of the VLO, many of the materials analyzed gave negative results in these aspects. As a conclusion, we can say that it is necessary for teachers in training and active teachers to participate in the design and development processes of the VLO so that the mistakes presented in the results of the analyzed materials can be corrected.
\end{abstract}

Key words: Virtual Learning Objects, ICT, Le Chatelier's Principle, Chemistry teaching, Analysis of Virtual Learning Objects.

\title{
1. INTRODUCCIÓN
}

Enseñar química no es una actividad sencilla, debido a que es una ciencia que integra conceptos (combustión, reacción química, estructura de Lewis) poco usuales en el lenguaje común, además son representados a través de ecuaciones químicas, símbolos químicos e iconemas; igualmente, en el proceso de enseñanza no se logra una articulación adecuada entre los tres niveles de representación (macroscópico, submicroscópico y simbólico) (Johnstone, 1982, Galagovsky, Rodríguez, Stamati \& Morales, 2003). En el caso particular de la enseñanza del principio de Le Chatelier, también es frecuente el uso de conceptos abstractos que exige a los estudiantes un dominio teórico de conceptos como: estequiometria, disoluciones, ley de los gases, concentración molar, reacciones químicas, concentración química, velocidad de reacción.

La mayoría de las actividades propuestas en el aula se basan en estrategias algorítmicas y descontextualizadas de la realidad, las prácticas de laboratorio empleadas por los profesores son casi nulas para hacer demostraciones en situaciones de equilibrio químico y las pocas que se hacen han recibido críticas y se han sugerido modificaciones (Raviolo \& Martínez, 2005), a esto se suma que la integración de las Tecnologías de la Información y la Comunicación (TIC) es casi nula por parte del profesor y la mayoría de estudiantes no las utilizan adecuadamente. Estos problemas generan dificultades en la comprensión del principio de Le Chatelier, por lo que es fundamental que el profesor busque alternativas que permitan que los estudiantes conceptualicen el tema y lo hagan propio. 
Domínguez Prada, E.D., Cabrera Castillo, H. G. (2020). Análisis de objetos virtuales de aprendizaje centrados en el Principio de Le Chatelier. Revista Convergencia Educativa, 7, julio, 1-21. DOI: http://doi.org/10.29035/rce.7.5

En este sentido, una de las alternativas que permite al profesor suplir estas necesidades de enseñanza de manera paulatina es la implementación progresiva de las TIC, que brinda muchas ventajas, entre ellas, ofrecen ambientes de aprendizaje que cautivan el interés del estudiante, igualmente, le otorgan libertad para explorar y construir conocimientos de acuerdo a sus necesidades, estimulan la creatividad y sentido crítico, y motiva al profesor a desarrollar innovaciones en la manera de plantear las clases (Ministerio de Educación Nacional, 2013, Díaz, 2013). Aclaramos que con la implementación de las TIC no se pretende reemplazar el papel que cumplen los profesores en el aula y tampoco se pretende dar solución a todos los problemas de enseñanza, aprendizaje y evaluación, sino que deben concebirse como un complemento que, vinculado con otras herramientas didácticas (material impreso, carteleras, afiches, material audiovisual), ayudaría a que el proceso de enseñanza se haga más asimilable para el estudiante.

Por lo anterior, los profesores de química deben aprovechar la gama de posibilidades que las TIC ofrecen; ejemplo de ello son los Objetos Virtuales de Aprendizaje (OVA) los cuales son recursos de carácter digital que pueden ser distribuidos y consultados en internet; se caracterizan por ser motivadores, facilitadores del aprendizaje y manejar un lenguaje hipermedial que permite que la información presentada se pueda manipular por medio de enlaces conectores. Los OVA hacen parte de los Materiales Educativos Computarizados (MEC), que se caracterizan por complementar lo que, por otros medios y materiales de enseñanza-aprendizaje, se dificulta lograr, tienen como objetivo apoyar el proceso de enseñanza aprendizaje, y son el modelo metodológico que se utiliza para el diseño y elaboración de los de los OVA (Galvis 1991, 1993, Silva \& Chica 2016).

Debido a la variedad de OVA que se pueden encontrar en la Web, surge una inquietud importante; si verdaderamente estos materiales que se pueden descargar e implementar dentro o fuera de las aulas de clase son apropiados para los objetivos que se deseen cumplir con ellos. Por tal razón, es necesario que estos sean evaluados por el profesor y posteriormente, adaptados a las estrategias de enseñanza, además, ser acordes a los objetivos que desee alcanzar con estos materiales. Por lo general, la selección de estos materiales se hace sin tener en cuenta aspectos técnicos, pedagógicos y de interactividad, de igual modo, es importante hacer la evaluación de estos materiales con el fin de conocer las ventajas y desventajas que pueda presentar en los aspectos de calidad de contenido, facilidad de uso, además de estar al tanto de las actualizaciones en el contenido que se presentan en el material (Galvis, 1993, Tabares, 2013, Bianchi, Saenz \& Rosanigo, 2008, Toll, Ruiz, Trujillo \& Rill, 2011). De acuerdo con lo anterior, el propósito que orientó este trabajo fue formular implicaciones a partir del análisis de OVA utilizados en la enseñanza del principio de Le Chatelier. 
Domínguez Prada, E.D., Cabrera Castillo, H. G. (2020). Análisis de objetos virtuales de aprendizaje centrados en el Principio de Le Chatelier. Revista Convergencia Educativa, 7, julio, 1-21. DOI: http://doi.org/10.29035/rce.7.5

\section{Algunas dificultades en la enseñanza-aprendizaje del principio de Le Chatelier}

El equilibrio químico y el principio de Le Chatelier, son temas considerados por los profesores de química como los que mayores dificultades presentan en el proceso de enseñanza aprendizaje (Narciso, Narciso \& Molina, 2015). La mayoría de las dificultades de aprendizaje que presentan los estudiantes al momento de estudiar el principio de Le Chatelier se derivan de la forma en que se les enseñó (Raviolo \& Andrade, 1998), destacándose la enseñanza bajo el modelo tradicional, que se caracteriza por el aprendizaje memorístico, en que la mayoría de los profesores se limitan a dictar el contenido del libro de texto, sin hacer la revisión de los errores conceptuales que estos puedan contener, y la mayoría de las veces se convierte en el único recurso didáctico de la clase (Quílez \& Sanjosé, 1995; Quílez \& Solaz, 1995), conjuntamente, la mayoría de los profesores presentan dificultades conceptuales similares a la de los estudiantes, como por ejemplo, confundir los conceptos de cantidad y concentración, separar los reactivos de los productos como sistemas aislados y tener una visión estática de los procesos de reacción química (Raviolo \& Martínez, 2003).

Muchas de estas problemáticas se derivan del poco uso de las TIC al momento de enseñar el principio de Le Chatelier; aún falta mucho para crear una cultura de uso e implementación de las TIC y el profesor debe estar a la vanguardia de las innovaciones tecnológicas para renovar los métodos de enseñanza y mejorar la comprensión de las problemáticas de enseñanza ya mencionadas, observar los diferentes niveles de representación de la materia (macroscópico, submicroscópico y simbólico) mediante simulaciones, comprender mejor un texto cuando este viene acompañado de animaciones, observar cómo afectan los estados de agregación, la velocidad de reacción, entre otros beneficios (Rodas, 2012, Camargo, 2014, Peña, 2014).

\subsection{Los objetos virtuales de aprendizaje}

En general los OVA, o también conocidos como Objetos de aprendizaje (OA) (Rojas, 2015), son definidos como recursos digitales autocontenidos y reutilizables, que cumplen un propósito educativo (Ministerio de Educación Nacional, 2013). Los OVA se pueden clasificar en imágenes, vídeos, animaciones, aplicaciones Web, cursos virtuales y software educativos (Ballesteros, Ricardo, Domínguez, Jiménez \& Morales, 2009).

En general, los OVA cuentan con la información de su contenido, denominado metadato, que facilita su almacenamiento y catalogación en Repositorios de Objetos de Aprendizaje (ROA), que son bibliotecas que contienen OVA a los que se puede acceder de forma gratuita. La búsqueda se hace mediante los metadatos del OVA. Los ROA deben contar con herramientas de búsqueda, es decir, que el usuario tenga la posibilidad de localizar, solicitar y recuperar el OVA de interés; los resultados arrojados por la búsqueda permiten a los usuarios previsualizar el OVA y, si lo desea, lo puede descargar (Astudillo, Sanz \& Willging, 2012). 
Domínguez Prada, E.D., Cabrera Castillo, H. G. (2020). Análisis de objetos virtuales de aprendizaje centrados en el Principio de Le Chatelier. Revista Convergencia Educativa, 7, julio, 1-21. DOI: http://doi.org/10.29035/rce.7.5

En el caso de la evaluación de los OVA, se pueden encontrar diversos instrumentos disponibles para ser utilizados por el profesorado de ciencias, especialmente los de química (Cova, Arrieta \& Aular, 2008, Obando \& Silva, 2013). De igual manera, Galvis $(1991,1993)$ diseñó seis formatos que incluyen juicio de expertos en contenido, metodología e informática. En ellos se hace la evaluación de criterios como la pertinencia del material, la viabilidad, interactividad, los objetivos del material, motivación, metodología e interfaz. De este modo, el evaluador tiene la posibilidad de resaltar los problemas del material, la localización del problema y hacer recomendaciones al respecto.

Tabla 1

Descripción de formatos de evaluación.

\begin{tabular}{|c|c|c|}
\hline Formato & Variable & Características \\
\hline \multirow[t]{4}{*}{$\begin{array}{l}\text { Descripción de Objeto } \\
\text { Virtual de Aprendizaje } \\
\text { (DMEC) }\end{array}$} & $\begin{array}{l}\text { Variables del entorno } \\
\text { OVA }\end{array}$ & $\begin{array}{l}\text { Aborda los aspectos de la población a la que se dirige el OVA, sus } \\
\text { necesidades educativas y equipo computacional para la instalación } \\
\text { del OVA. }\end{array}$ \\
\hline & Variables educativas & $\begin{array}{l}\text { Se evalúan los objetivos que se pueden alcanzar con el OVA, } \\
\text { aprendizajes previos, sistemas de motivación y refuerzo. }\end{array}$ \\
\hline & $\begin{array}{l}\text { Variables de } \\
\text { comunicación }\end{array}$ & $\begin{array}{l}\text { Se hace la evaluación de la interfaz de entrada y salida y las zonas } \\
\text { de comunicación. }\end{array}$ \\
\hline & $\begin{array}{l}\text { Variables de } \\
\text { computación }\end{array}$ & $\begin{array}{l}\text { Se evalúa si el OVA le permite al estudiante guardar el registro de las } \\
\text { secciones de trabajo y si le permite al profesor editar contenidos. }\end{array}$ \\
\hline $\begin{array}{l}\text { Valoración comprensiva } \\
\text { del Objeto Virtual de } \\
\text { Aprendizaje (EMEC-01) }\end{array}$ & Descripción general & $\begin{array}{l}\text { De forma general, se identifica el currículo al que pertenece el } \\
\text { OVA, evaluar si es interactivo y clasificar el tipo de software al } \\
\text { que pertenece (sistema tutorial, sistema de ejercitación, juego } \\
\text { educativo o simulador). }\end{array}$ \\
\hline \multirow{8}{*}{$\begin{array}{l}\text { Valoración de Objeto } \\
\text { Virtual de Aprendizaje, } \\
\text { enfoque de contenido } \\
\text { (EMEC-02) }\end{array}$} & Objetivos & $\begin{array}{l}\text { Se determina si los objetivos que plantea el material justifican ser } \\
\text { apoyados por un recurso digital como el OVA. }\end{array}$ \\
\hline & Contenido & Se determina si tiene coherencia con los objetivos. \\
\hline & $\begin{array}{l}\text { Desarrollo del } \\
\text { contenido }\end{array}$ & $\begin{array}{l}\text { Se verifica si la forma en que se presenta el contenido está } \\
\text { lógicamente organizada. }\end{array}$ \\
\hline & Micromundo & $\begin{array}{l}\text { Se determina si es acorde con el contenido, si ofrece al estudiante } \\
\text { situaciones excitantes o si , por el contrario, es aburrido y desligado } \\
\text { del contenido. }\end{array}$ \\
\hline & $\begin{array}{l}\text { Herramientas para } \\
\text { trabajar el micromundo }\end{array}$ & Se determina si las herramientas son sencillas de utilizar. \\
\hline & Ejemplos que ofrece & $\begin{array}{l}\text { Se evalúa si los ejemplos ilustran aspectos claves del contenido y } \\
\text { son suficientes para reforzar la información del contenido. }\end{array}$ \\
\hline & Ejercicios o retos & $\begin{array}{l}\text { Se evalúa si los ejercicios son variados y suficientes para que el } \\
\text { estudiante comprenda lo leído en el OVA. }\end{array}$ \\
\hline & Retro información & $\begin{array}{l}\text { Se determina si orienta al estudiante en la solución de los ejercicios, } \\
\text { brinda pistas o claves que permitan que el estudiante resuelva las } \\
\text { actividades. }\end{array}$ \\
\hline
\end{tabular}

Aunque los instrumentos mencionados previamente, contienen criterios de evaluación similares, los formatos propuestos por Galvis (1991) destacan la secuencialidad y articulación en que se aplican los instrumentos de evaluación, es decir, en cada uno de los formatos de evaluación se retoman aspectos que 
Domínguez Prada, E.D., Cabrera Castillo, H. G. (2020). Análisis de objetos virtuales de aprendizaje centrados en el Principio de Le Chatelier.

Revista Convergencia Educativa, 7, julio, 1-21. DOI: http://doi.org/10.29035/rce.7.5

en primera medida pudieron pasar desapercibidos, por ejemplo, los objetivos del material, la interactividad, los ejercicios, la metodología o el contenido (Tabla 1). Por condicionamientos de espacio, se omitió la información de los formatos relacionados con informática (Formato EMEC-04); valoración de objeto virtual de aprendizaje enfoque de metodología (Formato EMEC-03) y el formato final (Formato 5. Informe final evaluativo sobre OVA). Un aspecto fundamental que nos permitió decantarnos por estos formatos fue su apropiación en trabajos de investigación educativa, como los de Murillo \& Riascos (2011), Aponte \& Lárez (2014) y Baptista (2015).

\section{Aspectos metodológicos}

Este trabajo se elaboró bajo el enfoque cualitativo (Hernández, Fernández \& Baptista, 2010). Es decir, este enfoque fue fundamental para la descripción e interpretación de los resultados obtenidos (pertinencia, la viabilidad, interactividad y la calidad de los OVA seleccionados para el análisis) a partir de instrumentos aplicados a los OVA seleccionados que incluían el contenido conceptual del principio de Le Chatelier.

Los formatos de evaluación fueron aplicados a cinco OVA (Tabla 3), dicha muestra se obtuvo mediante la aplicación de criterios de búsqueda y criterios de selección, estos criterios se aplicaron con el fin de seleccionar OVA que fueran acordes al objetivo de este trabajo (Tabla 2). De este recorrido se obtuvieron un total de 14 OVA que cumplían con los criterios de búsqueda descritos. 
Domínguez Prada, E.D., Cabrera Castillo, H. G. (2020). Análisis de objetos virtuales de aprendizaje centrados en el Principio de Le Chatelier. Revista Convergencia Educativa, 7, julio, 1-21. DOI: http://doi.org/10.29035/rce.7.5

Tabla 2

Criterios de búsqueda y selección de OVA.

\section{Criterio de busqueda}

\begin{tabular}{|c|c|}
\hline Criterio & Descripción \\
\hline Contenido conceptual & $\begin{array}{l}\text { La busqueda en Google se centró en aquellos OVA que tuvieron como contenido principal el } \\
\text { principo de Le Chatelier o, en su defecto, que inluyera dicho principo en el contenido, por ello } \\
\text { se consideraron los OVA que incluyeran temas de cinética química y equilibrio químico. }\end{array}$ \\
\hline s de busqueda & $\begin{array}{l}\text { Las palabras de búsqueda introducidas en el buscador Google fueron: "Objetos virtuales de } \\
\text { aprendizaje equilibrio químico" y "Banco de objetos de aprendizaje". Mediante estas palabras } \\
\text { se buscaba restringir los resultados a materiales que cumplieran con las características de un } \\
\text { oVA. }\end{array}$ \\
\hline \multicolumn{2}{|r|}{ Criterio de selección } \\
\hline Criterio & Descripción \\
\hline OVA gratuitos & $\begin{array}{l}\text { Los OVA que se evaluen deben ser gratuitos, esto garantiza que un mayor número de usuarios } \\
\text { tengan acceso a estos materiales. }\end{array}$ \\
\hline $\begin{array}{l}\text { Informacion que lo } \\
\text { identifique }\end{array}$ & $\begin{array}{l}\text { Los OVA deben tener repositorios, donde se pueda consultar: nombre del autor, fecha de } \\
\text { elaboracion y objetivos. }\end{array}$ \\
\hline $\begin{array}{l}\text { Compatibilidad con los } \\
\text { sistemas operativos } \\
\text { habituales }\end{array}$ & Es importante que los OVA se adapten a los programas habituales: Linux o Windows. \\
\hline $\begin{array}{l}\text { Disponibilidad de } \\
\text { contenidos }\end{array}$ & $\begin{array}{l}\text { Algunos OVA no permiten acceder a la mayoria de sus contenidos, debido a que aún están } \\
\text { en proceso de desarrollo o son proyectados a futuro, por esto, los OVA a evaluar deben estar } \\
\text { totalmente terminados. }\end{array}$ \\
\hline
\end{tabular}

Después de aplicar los criterios de selección a los OVA, la muestra definitiva quedó reducida a 5 (ver Tabla 3) y fueron codificados del 1 al 5 para facilitar su referencia o mención en el trabajo.

Tabla 3

OVA para evaluar

\begin{tabular}{llll}
\hline Nombre del OVA & Repositorio & Dirección web & Código \\
\hline Cinética química & $\begin{array}{l}\text { Recursos libres. } \\
\text { Universidad } \\
\text { Autónoma de } \\
\text { Bucaramanga. }\end{array}$ & http://unabvirtual.edu.co/ovas/cinetica/ & OVA 1 \\
\hline $\begin{array}{l}\text { ¿En que se parecen } \\
\text { el equilibrio físico } \\
\text { y el equilibrio } \\
\text { químico? }\end{array}$ & $\begin{array}{l}\text { Centro de } \\
\text { innovación } \\
\text { educativa } \\
\text { regional sur. } \\
\text { Universidad del } \\
\text { Valle. }\end{array}$ & $\begin{array}{l}\text { http://objetos.ciersur.co/LO/S_G10_U04_L03/S_G10_U04_L03/ } \\
\text { index.html }\end{array}$ & OVA 2 \\
\hline $\begin{array}{l}\text { Equilibrio químico } \\
\text { Agrega 2 }\end{array}$ & $\begin{array}{l}\text { http://agrega.educacion.es/buscador2/BuscarAvanzadoCU/ } \\
\text { MostrarResultadosImagenesPrepararRetornoDetalleImagenes. } \\
\text { do?idioma=es\&buscContenido=equilibrio\%20 }\end{array}$ & OVA 3 \\
\hline $\begin{array}{l}\text { Equilibrio químico: } \\
\text { modificaciones del } \\
\text { estado de equilibrio }\end{array}$ & Agrega 2 & $\begin{array}{l}\text { http://agrega.juntadeandalucia.es/ } \\
\text { buscador2/DetallarODECU/DetallarODECU. } \\
\text { do?idioma=es\&identificadorODE=es-an_2010122213_9103105\& }\end{array}$ & OVA 4 \\
\hline $\begin{array}{l}\text { Chemical } \\
\text { equilibrium }\end{array}$ & $\begin{array}{l}\text { Gobierno de } \\
\text { Canarias }\end{array}$ & $\begin{array}{l}\text { http://education.uoit.ca/lordec/ID_LORDEC/chemistry_ } \\
\text { lechateliers/ }\end{array}$ & OVA 5 \\
\hline
\end{tabular}


Domínguez Prada, E.D., Cabrera Castillo, H. G. (2020). Análisis de objetos virtuales de aprendizaje centrados en el Principio de Le Chatelier. Revista Convergencia Educativa, 7, julio, 1-21. DOI: http://doi.org/10.29035/rce.7.5

\section{Resultados y análisis}

Con los cinco OVA seleccionados se procedió a aplicar cada uno de los formatos de evaluación indicados previamente. A continuación, se presentan los resultados de dicha evaluación de cada uno de los OVA.

\subsection{Descripción de objeto virtual de aprendizaje (FORMATO DMEC)}

Los cinco OVA analizados están dirigidos a estudiantes cuyas edades oscilan entre 16 y 18 años. Para el uso de estos materiales se requiere del uso de interfaces de entrada (ratón y teclado). El profesor puede trabajar los contenidos conceptuales como: cinética química, equilibrio químico, los factores que influyen en la velocidad de reacción, reacciones reversibles, constante de equilibrio, tipos de equilibrio, cociente de reacción, grado de disociación, reacciones de precipitación, relación del medio ambiente, la salud con el equilibrio químico y el principio de Le Chatelier. Los cinco materiales se puedan utilizar o instalar en los sistemas operativos computacionales convencionales (Windows, MacOS, Linux), asimismo, su descarga es gratuita, razón por la cual los estudiantes y comunidad en general lo pueden descargar y manipular desde cualquier lugar.

Es fundamental que los estudiantes, antes de trabajar con estos materiales, tengan claros conceptos como: concentración, estequiometría, nomenclatura, niveles de representación (macroscópico, submicroscópico y simbólico), diferenciar entre los conceptos calor y temperatura, concepto de mol, entalpía, entre otros, para que, de este modo, se centren en el aprendizaje del equilibrio químico y principio de Le Chatelier.

De acuerdo a lo anterior, es imprescindible que en el diseño de futuros OVA se involucren contenidos procedimentales y actitudinales con el propósito de promover en los estudiantes los planos de pensamiento personal significativo y relacional social, es decir, se debe avanzar hacia la producción de materiales basados en la experiencia de los estudiantes con el caso que se esté estudiando y a su vez, que permita el establecimiento de relaciones, colaboración y socialización orientadas hacia la resolución de problemas de diversa índole (Labarrere \& Quintanilla, 2002, Muñoz, Valencia, \& Cabrera-Castillo, 2017).

\subsection{Valoración comprensiva de objeto virtual de aprendizaje (FORMATO EMEC-01)}

El contenido de los cinco OVA hace parte del currículo de química y se centran en el equilibrio químico y el principio de Le Chatelier. Antes de la aplicación de cada OVA es importante que los estudiantes hagan un recorrido previo por el material con el fin de conocer los comandos que tiene a disposición, por ejemplo, si 
Domínguez Prada, E.D., Cabrera Castillo, H. G. (2020). Análisis de objetos virtuales de aprendizaje centrados en el Principio de Le Chatelier. Revista Convergencia Educativa, 7, julio, 1-21. DOI: http://doi.org/10.29035/rce.7.5

permite subir o bajar el volumen de la música de fondo o de los efectos sonoros, tener la oportunidad de reiniciar alguna actividad o guardar alguna sección.

En cuanto a la interactividad, los OVA 1 y 4 presentan limitaciones para el estudiante, debido a que se centran en brindar información teórica y hacer la evaluación de esta, mediante preguntas de selección múltiple, dejando de lado otro de tipo de actividades que capten su atención, por ejemplo, situaciones problema o preguntas abiertas que fomenten el debate en clase con los compañeros y el profesor (Márquez \& Roca, 2006, Roca, Márquez \& Sanmartí, 2013), tampoco se hace inclusión de prácticas experimentales en las que los estudiantes puedan comprender de forma real algunos sucesos del principio de Le Chatelier. De acuerdo con Galvis (1991), estos OVA se catalogan como sistemas de ejercitación y práctica, por lo tanto, deben tener variedad de ejercicios y contar con retroalimentación que oriente al usuario en la búsqueda del aprendizaje, por ello, no es pertinente que un OVA se limite a decir al usuario frases como "inténtalo de nuevo" o, simplemente, que se pueda acceder fácilmente a la respuesta de la pregunta planteada. Este tipo de situaciones no garantizan que el estudiante, se motive a utilizar el OVA.

Lo contrario sucede con los OVA 2 y 5, que sí cuentan con variedad de ejercicios. En el caso del OVA 2, cuenta con diferentes formatos para la presentación de las situaciones problema como la historieta, las preguntas abiertas, resúmenes, laboratorios y vídeos. El OVA 5 cuenta con una sección de evaluación donde se resumen todos los temas del material y el estudiante debe responder una serie de preguntas de variado nivel. El OVA 3 se clasificó como un sistema de simulación para explorar, en el cual el estudiante tiene la posibilidad de interactuar con cada una de las simulaciones y hacer observación de cada situación. Según Galvis (1991), Contreras, García \& Ramírez (2010) y Talanquer (2014) las simulaciones se definen como programas que contienen un modelo de algún aspecto del mundo y que permite al estudiante cambiar ciertos parámetros o variables de entrada; son de fácil instalación y manipulación, cumplen una función motivadora ya que la mayoría de los estudiantes se sienten atraídos por este tipo de programas y satisfacen una función de apoyo para el profesor.

\subsection{Valoración de objeto virtual de aprendizaje enfoque de contenido (FORMATO EMEC-02)}

Para la valoración de los OVA, se hizo énfasis en los objetivos, el contenido, su desarrollo, el micromundo (área de trabajo del OVA), las herramientas para trabajar el micromundo, los ejemplos que ofrece, los ejercicios o retos y la retro información.

Iniciemos con los objetivos, el OVA 1 se formula tres: "comprender el significado de cinética química", "identificar las teorías que sustentan la cinética química” y "reconocer los factores que afectan la velocidad de las reacciones". Dichos objetivos no se pueden alcanzar con las actividades propuestas por el material ya 
Domínguez Prada, E.D., Cabrera Castillo, H. G. (2020). Análisis de objetos virtuales de aprendizaje centrados en el Principio de Le Chatelier. Revista Convergencia Educativa, 7, julio, 1-21. DOI: http://doi.org/10.29035/rce.7.5

que son insuficientes y no son variadas, debido a que solo se formulan preguntas de selección múltiple y no implementa situaciones problema. En el caso del OVA 2, el único objetivo planteado fue "analizar en términos energéticos y cinéticos las reacciones químicas reversibles"; para alcanzarlo, el OVA hace uso de experimentos, videos demostrativos y narración de sucesos en formato de historieta. Esta última, es considerada como un medio que resulta beneficioso para el proceso de enseñanza-aprendizaje de cualquier materia, ya que fomenta la creatividad, motivación y adquisición de conocimiento, además, los estudiantes se identifican con los sucesos narrados y se mantiene el equilibrio entre la fantasía y la realidad (Baudet, 2011, Misrachi \& Alliende, 1994).

Algunos de los objetivos planteados por el OVA 3 son: "entender la importancia de la ley de acción de masas", "relación de la constante de equilibro" y "la energía de Gibbs". Para alcanzar dichos objetivos el OVA hace uso de simulaciones, pero hace falta incluir contenidos (conceptuales, actitudinales y procedimentales) que orienten al estudiante en el proceso de aprendizaje. En el caso del OVA 4, los objetivos planteados son: “que el estudiante pueda establecer en qué momento una reacción está en equilibrio y, de no estarlo, establecer el sentido de la reacción"; "que el estudiante comprenda cuales son los factores que pueden alterar el equilibrio de una reacción"; este OVA se podría catalogar como un libro de texto digital que se caracteriza por la implementación de contenidos audiovisuales, interactividad, y la personalización y la actualización (Sotomayor, 2015), sin embargo el OVA 4 no cuenta con interactividad para el estudiante puesto que solo se presenta información teórica y actividades, cuyas respuestas pueden ser consultadas fácilmente, por ende, no es posible que el estudiante pueda alcanzar dichos objetivos. El OVA 5, plantea los siguientes objetivos: "comprender los cambios producidos por la temperatura", "comprender los cambios producidos por la presión/volumen", “comprender los cambios producidos por la concentración”. Para alcanzar dichos objetivos el OVA cuenta con variedad de ejercicios para el estudiante y, al final del OVA, se plantea una evaluación que retoma los temas vistos, con el fin de evaluar lo aprendido por el estudiante.

De acuerdo a la descripción anterior se pudo establecer que todos los objetivos planteados por cada uno de los OVA vale la pena que se apoyen con las TIC, desde la enseñanza de las leyes que rigen el principio de Le Chatelier hasta su aplicación o desarrollo en la vida cotidiana, permitiendo, no solo al estudiante, adquirir estrategias de aprendizaje y desarrollo de competencias instrumentales, interpersonales y sistemáticas (Martínez, Bonet, Cáceres, Fargueta \& García, 2007), también favorece que el profesor se haga partícipe de la implementación de estos recursos y no se sienta ajeno a su uso, pero hay que dejar claro que la información suministrada por estos materiales debe ser complementada por el profesor, para evitar vacíos conceptuales.

En cuanto al contenido y su desarrollo, los OVA 1, 2, 4 y 5 son coherentes con el contenido que plantean y los objetivos, es decir, a medida que se avanza en la lectura del contenido de cada OVA se puede observar 
Domínguez Prada, E.D., Cabrera Castillo, H. G. (2020). Análisis de objetos virtuales de aprendizaje centrados en el Principio de Le Chatelier. Revista Convergencia Educativa, 7, julio, 1-21. DOI: http://doi.org/10.29035/rce.7.5

cómo los objetivos se retoman ya sea en el planteamiento de actividades, ejemplos o simplemente información teórica. Sin embargo, el contenido de los OVA 1 y 3 no es suficiente para alcanzar los objetivos propuestos en cada material, en el caso del OVA 1, el contenido se limita a brindar información teórica a los estudiantes, pero no se hace una profundización de cada tema. El OVA 3 aunque cuenta con simulaciones para representar cada uno de los temas, no tiene suficiente información teórica para el estudiante, por tanto, este OVA solo servirá como un recurso para practicar lo aprendido o leído en otro tipo de materiales. Para los OVA 2, 4 y 5 la información teórica sí es suficiente para lograr los objetivos propuestos, debido a que tienen suficiente información teórica para resolver los ejercicios planteados, aun así, es necesario que se complemente con las clases magistrales.

En el caso del OVA 3, se pudo establecer que para el objetivo "Entender el interés que la ley de acción de masas tuvo como origen de la formalización actual del equilibrio químico", se puede inferir que este objetivo busca que el estudiante comprenda desde el origen histórico, la importancia de la ley de acción masas para el equilibrio químico, sin embargo, al dirigirse a la unidad de "la constante de equilibrio", donde se aborda este término, no hay una referencia teórica al respecto. Pero sí se hace en el material de apoyo del OVA que está en formato PDF, por lo que es necesario que el OVA indique al estudiante que este objetivo se amplía en el material de apoyo, y no dentro del OVA.

Es importante dejar claro que la información teórica, si se presenta de forma reducida, debe ser lo suficientemente clara para el estudiante y estar organizada de acuerdo con los objetivos del OVA y permitir la navegación del usuario, guiándolo en la comprensión y construcción del conocimiento; los elementos deben estar rotulados o etiquetados para especificar los contenidos, las actividades y los elementos de contextualización, con los términos apropiados (Morales, Gutiérrez, \& Ariza, 2016).

Para los OVA 1, 2, 3, 4 y 5 el desarrollo del contenido es claro para el estudiante, es decir, la terminología utilizada por estos OVA es comprensible para los estudiantes ya que no hay conceptos que no se hayan trabajado previamente a lo largo de las clases de química, por ejemplo, el OVA 2 cuando menciona el termino hipoxia, brinda el significado al estudiante inmediatamente, lo mismo sucede con el OVA 3, que explica cómo se calcula el cociente de reacción Q. En todos los OVA el estudiante sabe en qué parte del contenido se encuentra. Cada uno de los OVA tiene la forma de indicarle al estudiante en qué capítulo se encuentra, ya sea mediante los nombres de los capítulos, o resaltando el nombre con colores llamativos en el menú.

Es necesario que los diseñadores como los desarrolladores de OVA hagan énfasis en el contenido de estos, no tiene sentido trascribir el contenido de un libro de texto a un formato digital; lo ideal es hacer una transposición del contenido científico para que sea accesible a los estudiantes, por lo que es importante tener claro el tipo de estudiantes al que se va a dirigir el OVA (estudiantes con condiciones de aprendizaje 
Domínguez Prada, E.D., Cabrera Castillo, H. G. (2020). Análisis de objetos virtuales de aprendizaje centrados en el Principio de Le Chatelier. Revista Convergencia Educativa, 7, julio, 1-21. DOI: http://doi.org/10.29035/rce.7.5

especiales, con déficit de atención) asimismo, el contenido debe estar actualizado con el currículo que rija en el momento en que se esté diseñando el material (Escudero, 2003).

El micromundo de los 5 OVA es de tipo gráfico, solo el OVA 1 cuenta con narración del contenido en las primeras páginas de cada unidad, sin embargo, el micromundo de este OVA no tiene significado para el estudiante ya que la única opción que le brinda a los estudiantes es elegir opciones de respuesta a las preguntas planteadas, lo mismo sucede con el OVA 4 que, aunque incorpora vídeos y simulaciones, no son suficientes ya que el mismo material se encarga de suministrar las respuestas de las preguntas a los estudiantes, quitando la posibilidad de que ellos exploren y busquen la respuesta de dichas preguntas, además, los vídeos están en inglés. En síntesis, el micromundo de estos OVA no permite al estudiante enfrentar situaciones excitantes o de variado nivel como juegos alusivos al principio de Le Chatelier o problemas de la vida cotidiana donde se involucre el equilibrio químico.

El micromundo de los OVA 2, 3 y 5 sí tienen relevancia para el estudiante y mantienen coherencia con el contenido y, aunque el OVA 2 no presenta muchas situaciones en las que el estudiante pueda observar lo ocurrido, cuando se le da una orden al OVA (por ejemplo, cambiar una variable, o que algún personaje se mueva), se complementa con el tipo de actividades propuestas por el material, como los vídeos que incluyen simulaciones demostrativas, que además plantean preguntas problematizadoras, de igual modo, plantea laboratorios que permiten que los estudiantes trabajen en la clase o en casa. Para los OVA 3 y 5, los estudiantes pueden manipular simulaciones y ejemplos propuestos, que permiten tomar decisiones, analizar casos, y dar solución al problema planteado (Galvis, 1992, Alava, Aguirre, Cabrera, Campaña \& Maya, 2011). Asimismo, estos micromundos permiten que el estudiante enfrente situaciones excitantes o de variado nivel, ya que incluye diversos ejemplos, ejercicios y los formatos en que se plantean dichas actividades también es variado.

Para los OVA 1, 4 y 5, las herramientas del micromundo no cuentan con ayudas de utilización, debido a que los comandos (subir el volumen, cambiar de página, recargar página, iniciar sección) no tienen un indicador escrito que muestre al estudiante la función de dicho comando. Lo contrario sucede con los OVA 2 y 3 , que además de tener los íconos de representación, indican al estudiante de manera escrita la función del comando, facilitando su navegación por el OVA, por tanto, si un estudiante que no ha tenido experiencias con las TIC, tendría dificultades para identificar los íconos que representan cada función.

Los ejemplos propuestos en los OVA 1, 3 y 4 son insuficientes para el estudiante, debido a que son escasos y no ilustran aspectos relevantes del contenido, por ejemplo, el contenido del OVA 4, aunque incluye vídeos y simulaciones como herramientas para plantear problemas, no cumple dicha función de evaluación, ya que, como se ha venido reiterando, estas actividades tienen incorporada la respuesta de las preguntas, por tanto, 
Domínguez Prada, E.D., Cabrera Castillo, H. G. (2020). Análisis de objetos virtuales de aprendizaje centrados en el Principio de Le Chatelier. Revista Convergencia Educativa, 7, julio, 1-21. DOI: http://doi.org/10.29035/rce.7.5

pasan a cumplir el papel de ejemplificar el contenido, lo mismo sucede con el OVA 1, cuyas preguntas de selección múltiple no hacen un aporte significativo al proceso de aprendizaje del estudiante.

Los OVA 2 y 5 tienen variedad de ejemplos y son relevantes para el estudiante, por ejemplo, en el OVA 2 se contextualizan los conceptos de temperatura, presión y velocidad de reacción con situaciones cotidianas, por ejemplo, el efecto de la temperatura en ciertos medicamentos, el efecto de la velocidad de reacción en procesos metabólicos del cuerpo humano; con dichos ejemplos el estudiante se siente identificado y favorecen la comprensión de estos conceptos de mejor manera.

De acuerdo a lo anterior, es indispensable que los OVA, tendientes a ser desarrollados en el futuro, cuenten con variedad de ejemplos, tal como el formato en que se presenten. El análisis de los OVA arrojó que la mayoría utiliza ejemplos escritos, o basados en imágenes. Estos formatos cumplen diferentes funciones tales como describir situaciones o fenómenos; es necesario dirigir al estudiante mediante palabras o tareas específicas, en cuanto a la lectura de las imágenes para, de esta forma, optimizar el efecto sobre el aprendizaje, puesto que, no todos tienen la misma capacidad para utilizar la información gráfica (Perales \& Jiménez, 2002). Hay que tener en cuenta que las imágenes que se utilicen deben apoyar al lector en la lectura, guiarlo, y no cumplir solo una función estética, como ocurre con los OVA 1, 3 y 4.

En cuanto a los ejercicios o retos los OVA 1 y 4, plantean ejercicios que permiten comprobar el dominio de los objetivos por parte del estudiante, pero los formatos en que se plantean dichos ejercicios no son variados, ya que solo se plantean preguntas de selección múltiple y, en el caso del OVA 4, dichas preguntas tienen la respuesta que puede ser consultada fácilmente. Los OVA 2, 3 y 5 sí tienen múltiples formatos de proponer las preguntas; estos plantean preguntas abiertas, interpretación de gráficas, experimentos, preguntas de selección múltiple y son suficientes para que el estudiante compruebe los conocimientos adquiridos en cada material.

Se pudo establecer que los cinco OVA descritos se enfocan en plantear preguntas de selección múltiple con única respuesta o preguntas abiertas, pero no se plantean otro tipo de preguntas; de acuerdo a la literatura, hay otros tipos de preguntas como, la pregunta mediadora, que tiene como finalidad posibilitar y favorecer que el estudiante se situé en una de las diversas disciplinas desde la cual puede abordar o dar solución a un fenómeno en particular, por consiguiente debe plantear de manera explícita la interacción entre el sistema (adentro), su ambiente (fuera), considerar la regulación de este (orden-desorden) y su orientación temporal (estabilidad-cambio), pero también deben tomar como referencia los niveles de organización de la materia macroscópico y microscópico (Márquez \& Roca, 2006). También están las preguntas memorísticas, en la que el estudiante debe recordar de forma clara la información y preguntas de traslación, exige que el estudiante transforme la información en un lenguaje diferente sin cambiar el su sentido original (Morón, 2015). 
Domínguez Prada, E.D., Cabrera Castillo, H. G. (2020). Análisis de objetos virtuales de aprendizaje centrados en el Principio de Le Chatelier. Revista Convergencia Educativa, 7, julio, 1-21. DOI: http://doi.org/10.29035/rce.7.5

En cuanto a la retroalimentación, los OVA 1 y 3 no indican al estudiante las razones por las que una respuesta es correcta o incorrecta, más bien, ante el fallo de una respuesta estos OVA se limitan a decir al estudiante las siguientes frases: "incorrecto", "mal", o "correcto", "muy bien sigue así". En el caso del OVA 1, el estudiante cuenta con tres oportunidades para responder a la pregunta, y si luego de estos intentos no se acierta con la respuesta, automáticamente se pasa al siguiente ejercicio. Para el OVA 3, aunque en algunos casos sí indica al estudiante las razones por las que la respuesta es incorrecta, brinda la respuesta completa a la pregunta, según Galvis (1991), este tipo de acciones son contraproducentes para el estudiante ya que limitan la posibilidad de que el estudiante piense para llegar a la respuesta. Aunque la mayoría de las preguntas del OVA 2 son abiertas, sí cuenta con dos actividades para ser resueltas por el estudiante dentro del material y, al igual que los OVA anteriores, la retroalimentación no indica al estudiante las razones por las que las respuestas son correctas o incorrectas, en este caso, el profesor sería quien complementa esta acción.

Los OVA 4 y 5 sí le indican al estudiante las razones de porqué una respuesta es correcta o incorrecta, por ejemplo, en el OVA 5, si la respuesta es correcta, lo indica al estudiante con la frase "correcto" y, además, se hace la retroalimentación según el tema de la pregunta, por ejemplo, "correcto, el equilibrio se desplaza hacia la derecha para aumentar la temperatura".

\section{Implicaciones del análisis de OVA}

Es fundamental promover una motivación intrínseca que se caracteriza por proponer ambientes o situaciones amigables e interesantes, que despiertan curiosidad en el alumno de modo que se fomente la indagación Galvis (1991); la forma en que se presentan las situaciones problema es en forma de historieta; por medio de preguntas abiertas, motivar al estudiante a hacer indagaciones y proponer soluciones profundas.

El papel del estudiante respecto de estos materiales debe ser tan activo como si se tratara de una clase presencial; estos materiales deben garantizar la interacción entre todos, el alumno debe concebir el OVA como un material relevante que hace un aporte significativo el aprendizaje, no solo se trata de que sean asequibles, sino que también cumplan con las expectativas del estudiante y el profesor (Bryndum \& Montes, 2005). Se considera al estudiante como poseedor de una estructura cognitiva que soporta el proceso de aprendizaje, en síntesis, se tienen en cuenta la integración de los conceptos científicos que el estudiante pueda lograr (Ruiz, 2007).

Es necesario tener en cuenta que la metodología que contenga el OVA influirá positiva o negativamente en la motivación del estudiante pues, no es lo mismo tener un OVA cargado de texto, en comparación con 
Domínguez Prada, E.D., Cabrera Castillo, H. G. (2020). Análisis de objetos virtuales de aprendizaje centrados en el Principio de Le Chatelier.

Revista Convergencia Educativa, 7, julio, 1-21. DOI: http://doi.org/10.29035/rce.7.5

una cuya información sea reducida pero concreta y con actividades que fomenten el trabajo en grupo, por esta razón es necesario que los profesores se hagan participes del diseño de este tipo de materiales y de los procesos de desarrollo de los OVA; en los materiales analizados se pudo establecer que muchos de estos presentan dificultades en el uso de la metodología y el contenido; esto se hace evidente por la forma de plantear las preguntas y las actividades, además, se satura al estudiante con mucha información, dejando de lado el espacio para la elaboración de actividades que permitan comprobar lo aprendido, no solo dentro del OVA, sino también en el aula.

Es importante que el profesor seleccione un OVA interactivo para el estudiante que incluya en su contenido simuladores, vídeos, laboratorios, situaciones problema. No es conveniente que los estudiantes interactúen con un OVA que se limite a hacer la presentación del contenido de forma lineal, como lo hacen la mayoría de los libros de texto.

Aunque un OVA, aparentemente, sea elemental, si se utiliza con una estrategia de enseñanza bien diseñada, puede dar buenos resultados, por esto, que debe ser incluido dentro de la planeación de la clase para evaluar en qué momento es oportuno utilizarlos, iniciando la clase, a mitad sesión o al final y, asimismo, tener en cuenta el rol que ocupará en la clase, si será útil para ejemplificar algunos temas o para que el estudiante practique con los ejercicios planteados por cada material.

\section{Conclusiones}

Las implicaciones que se derivaron del análisis de los OVA demuestran que hace falta que los profesores tengan en cuenta criterios de evaluación como la metodología que el material utiliza, la interactividad, el contenido, los objetivos; por esta razón es pertinente que el profesor esté a la vanguardia de las innovaciones de las TIC, que incluye a los OVA y otros tipos de materiales digitales que sirven de herramientas para el proceso de enseñanza-aprendizaje.

Es importante que los profesores en formación y los activos, aprovechen las múltiples bondades que estos recursos pueden ofrecer, pero también es necesario que se haga una constante evaluación de los OVA.

Se espera que los resultados de esta investigación sean tenidos en cuenta por los diseñadores, desarrolladores y usuarios de OVA, para no repetir las falencias encontradas en estos materiales y se tengan herramientas para reconocer un material de calidad que se ajuste a las necesidades educativas y no solo llevar al aula materiales que solo cumplan la función de entretener a los estudiantes sin hacer un aporte significativo al proceso de enseñanza-aprendizaje. 
Domínguez Prada, E.D., Cabrera Castillo, H. G. (2020). Análisis de objetos virtuales de aprendizaje centrados en el Principio de Le Chatelier.

Revista Convergencia Educativa, 7, julio, 1-21. DOI: http://doi.org/10.29035/rce.7.5

Se encontró que, de los cinco OVA analizados, solo el OVA 2 cumple con las características de calidad como: metodología, contenido e interactividad; los OVA 3 y 5 tuvieron una calificación aceptable. En cuanto a los OVA 1 y 4, se encontraron varias falencias como, por ejemplo, que no son interactivos, el contenido no es suficiente para alcanzar los objetivos propuestos, entre otros. Estos aspectos deben ser tenidos en cuenta para ser corregidos en desarrollos futuro.

\section{REFERENCIAS BIBLIOGRÁFICAS}

Alava, C., Aguirre, A., Cabrera, H., Campaña, S., \& Maya, J. (2011). Creación de micromundos aplicando la teoría de juegos y el diseño orientado a objetos. Revista de investigaciones UNAD, 10(1), 1-11. Recuperado de https://academia.unad.edu.co/images/investigacion/hemeroteca/revistainvestigaciones/volumen10num1\%202011/13.\%20Creacion\%20de\%20micromundos\%20aplicando.pdf

Aponte, A., \& Lárez, N. (2014). Material Educativo Computarizado para el aprendizaje del contenido de Estequiometria de Reacciones Químicas en la asignatura de Química General I (Tesis de pregrado). Universidad de Carabobo, Venezuela. Recuperado de http://riuc.bc.uc.edu.ve/bitstream/123456789/1504/3/4599.pdf

Astudillo, G., Sanz, C., \& Willging, P. (2012). Repositorios de objetos de aprendizaje: un espacio de búsqueda centrado en lo educativo. En VII Congreso de Tecnología en Educación y Educación en Tecnología. Universidad Nacional de La Plata, Argentina. Recuperado de http://sedici.unlp.edu.ar/handle/10915/18494

Ballesteros, B., Ricardo, C., Domínguez, E., Jiménez, E., \& Morales, G. (Ed.). (2009). Mitos de los objetos de aprendizaje y realidades de la experiencia de la universidad del Norte. En V. Claudia y J. Alexa (Ed.), Objetos de aprendizaje prácticas y perspectivas educativas (pp. 97-111). Santiago de Cali: Colección Univirtual.

Baptista, R. (2015). Material educativo computarizado: una herramienta tecnológica educativa, previa a la Práctica I del laboratorio de Química (Tesis de Maestría). Universidad de Carabobo, Venezuela.

Baudet, J. (2011). La historieta como medio para la enseñanza. (Tesis de pregrado). Universidad Católica Andrés Bello, Caracas, Venezuela.

Bianchi, G., Saenz, M., \& Rosanigo, Z. (2008). Objetos de aprendizaje: análisis de la calidad. Recuperado de https://pdfs.semanticscholar.org/5796/b1be950f4ca0b3738a290d1aed883a328df3.pdf 
Domínguez Prada, E.D., Cabrera Castillo, H. G. (2020). Análisis de objetos virtuales de aprendizaje centrados en el Principio de Le Chatelier.

Revista Convergencia Educativa, 7, julio, 1-21. DOI: http://doi.org/10.29035/rce.7.5

Bryndum, S., \& Montes, J. (2005). La motivación en los entornos telemáticos. RED. Revista de educación a distancia, 13, 1-24. Recuperado de https://www.um.es/ead/red/13/bryndum.pdf

Camargo, P. (2014). Las TIC como herramientas facilitadoras en la gestión pedagógica. Boletín Informativo Edición 6. Recuperado de https://www.utb.edu.co/newsletter/educacionadistancia/2014/bole$\underline{\text { tin006/noti apliaciones/005-lastic/index.html }}$

Contreras, G., García, R., \& Ramírez, M. (2010). Uso de simuladores como recurso digital para la transferencia de conocimiento. Apertura, Revista de innovación educativa, 2(1), 86-100. Recuperado de https://dialnet.unirioja.es/servlet/articulo?codigo=5547092

Cova, A., Arrieta, X., \& Aular, J. (2008). Revisión de modelos para evaluación de software educativo. Revista electrónica de estudios telemáticos TÉLÉMATIQUE, 7(1), 1-24. Recuperado de http://ojs.urbe.edu/index.php/telematique/article/view/1969

Díaz, D. (2013). TIC en Educación Superior: ventajas y desventajas. Educación y Tecnología, (4), 44-50. Recuperado de http://revistas.umce.cl/index.php/edytec/article/view/180

Escudero, T. (2003). La formación pedagógica del profesorado universitario vista desde la enseñanza disciplinar. Revista de educación, 331, 101-121. Recuperado de https://books.google.cl/books?id=]XtfB$\underline{\mathrm{wAAQBA}} \&$ printsec $=$ frontcover $\& \mathrm{hl}=\mathrm{es} \# \mathrm{v}=$ onepage $\& \mathrm{q} \& \mathrm{f}=$ false

Galagovsky, L., Rodríguez, M., Stamati, N., \& Morales, L. (2003). Representaciones mentales, lenguajes y códigos en la enseñanza de ciencias naturales. Un ejemplo para el aprendizaje del concepto de "reacción química" a partir del concepto de "mezcla". Enseñanza de las ciencias: revista de investigación y experiencias didácticas, 21(1), 107-121.

Galvis, A. (1991). Ingeniería de software educativo, versión 3. Bogotá: Universidad de los Andes.

Galvis, A. (1992). Ingeniería de Software Educativo. Bogotá: Uniandes. Recuperado de https://es.slideshare.net/algalvis50/ise1992-parte-0-inicio-y-fin

Galvis, A. (1993). Evaluación de materiales y ambientes educativos computarizados. Informática educativa, 6(1), 9-27. Recuperado de http://rie.uniandes.edu.co/LinkClick.aspx?fileticket=yHTc4CagzkQ\%3D\&amp;tabid=432\&amp;mid=1385\&amp;forcedownload=true=\%3C!--19930611evaluac.pdf--\%3E\%3Ca\%20href=\%22/LinkClick.aspx?fileticket=yHTc4CagzkQ\%3D\&amp;tabid=432\&amp;mid=1385\&amp;forcedownload=true $\% 22 \% 20 \% 20$ target $=\% 22$ blank $\% 22 \% 3 \mathrm{E} 19930611$ evaluac.pdf $\% 3 \mathrm{C} / \mathrm{a} \% 3 \mathrm{E}$

Hernández, R., Fernández, C., \& Baptista, P. (2010). Metodología de la investigación. México: McGraw-Hill. 
Domínguez Prada, E.D., Cabrera Castillo, H. G. (2020). Análisis de objetos virtuales de aprendizaje centrados en el Principio de Le Chatelier.

Revista Convergencia Educativa, 7, julio, 1-21. DOI: http://doi.org/10.29035/rce.7.5

Johnstone, A. H. (1982). Macro- and microchemistry. School Science Review, 64(277), 377-379.

Labarrere, A., \& Quintanilla, M. (2002). La solución de problemas científicos en el aula. Reflexiones desde los planos de análisis y desarrollo. Pensamiento Educativo. Revista de Investigación Educacional Latinoamericana, 30(1), 121-137. Recuperado de http://pensamientoeducativo.uc.cl/index.php/pel\%20/article/view/216

Márquez, C., \& Roca, M. (2006). Plantear preguntas: un punto de partida para aprender ciencias. Revista Educación y Pedagogía, 18(45), 61-71. Recuperado de https://revistas.udea.edu.co/index.php/revistaeyp/article/view/6087

Martínez, S., Bonet, P., Cáceres, P., Fargueta, F., \& García, E. (2007). Los objetos de aprendizaje como recurso de calidad para la docencia: criterios de validación de objetos en la Universidad Politécnica de Valencia., TICAI2007: TICs para el Aprendizaje de la Ingeniería, 67-72. Recuperado de http://ceurws.org/Vol-318/Naharro.pdf

Ministerio de Educación Nacional. (2013). Competencias TIC para el desarrollo profesional docente. Recuperado de https://www.mineducacion.gov.co/1759/articles-339097 archivo pdf competencias tic.pdf

Misrachi, C., \& Alliende, F. (1994). La historieta como medio educativo y como material de lectura. Lectura $y \quad$ vida. 12, 1-13. Recuperado de http://www.lecturayvida.fahce.unlp.edu.ar/numeros/a12n3/12 03 Misrachi.pdf

Morales, L., Gutiérrez, L., \& Ariza, L. (2016). Guiá para el diseño de objetos virtuales de aprendizaje (OVA). Aplicación al proceso enseñanza-aprendizaje del área bajo la curva de cálculo integral. Revista científica General José María Córdova, 14(18), 127-147. Recuperado de https://www.redalyc.org/pdf/4762/476255360008.pdf

Morón, F. (2015). La importancia de hacer buenas preguntas a nuestros alumnos de la ESO. Artista Digital, 54, 1-12. Recuperado de http://www.afapna.es/web/aristadigital/archivos revista/2015 marzo 5.pdf

Murillo, E., \& Riascos, E. (2011). Evaluación y selección de un material educativo computarizado para la enseñanza del sistema digestivo (Tesis de pregrado). Universidad del Valle, Buenaventura, Colombia.

Muñoz, F., Valencia, E., \& Cabrera-Castillo, H. G. (2017). Situaciones científicas escolares problematizadoras a partir del análisis del experimento V de Robert Boyle. Revista Eureka sobre Enseñanza y 
Domínguez Prada, E.D., Cabrera Castillo, H. G. (2020). Análisis de objetos virtuales de aprendizaje centrados en el Principio de Le Chatelier.

Revista Convergencia Educativa, 7, julio, 1-21. DOI: http://doi.org/10.29035/rce.7.5

Divulgación de las Ciencias, 14(1), 115-125. Recuperado de https://www.redalyc.org/pdf/920/92049699009.pdf

Narciso, P., Narciso, J., \& Molina, J. (2015). Los simuladores como recurso educativo ante las consideraciones alternativas del Equilibrio Químico en estudiantes universitarios. Recuperado de https://web.ua.es/es/ice/jornadas-redes-2015/documentos/tema-1/410893.pdf

Obando, J., \& Silva, M. (2013). Un Objeto Virtual de Aprendizaje (OVA) para el curso de física mecánica. Memorias, 11(19), 125-134. Recuperado de https://pdfs.semanticscholar.org/68e8/cb98894198a3f949210d65cffc90f6b36f6f.pdf

Peña, J. (2014). Análisis comparativo en el uso de las TIC para aplicaciones educativas de la competencia tecnológica. TLATEMOANI, Revista Académica de Investigación, 15, 1-17. Recuperado de https://materialdidacticontics.files.wordpress.com/2016/05/revista-6.pdf

Perales, F., \& Jiménez, J. (2002). Las ilustraciones en la enseñanza-aprendizaje de las ciencias. Análisis de libros de texto. Enseñanza de las Ciencias, 20(3), 369-386. Recuperado de https://core.ac.uk/reader/13268068

Quílez, P., \& Sanjosé, L. (1995). Errores conceptuales en el estudio del equilibrio químico: nuevas aportaciones relacionadas con la incorrecta aplicación del principio de Le Chatelier. Enseñanza de las Ciencias, 13(1), 72-80. Recuperado de https://core.ac.uk/download/pdf/38990402.pdf

Quílez, P., \& Solaz, P. (1995). Students' and teachers' misapplication of le chatelier's principle: Implications for the teaching of chemical equilibrium. Journal of research in science teaching, 32(9), 939-957. DOI: https://doi.org/10.1002/tea.3660320906

Raviolo, A., \& Andrade, J. (1998). Enseñar el principio de Le Chatelier. Un sutil equilibrio. Educación Química, 9(1), 40-45. DOI: http://dx.doi.org/10.22201/fq.18708404e.1998.1.66587

Raviolo A., \& Martínez, M. (2005). El origen de las dificultades y de las concepciones alternativas de los alumnos en relación con el equilibrio químico. Educación Química, 16(4e), 159-166. DOI: http://dx.doi.org/10.22201/fq.18708404e.2005.4e.66080

Raviolo, A., \& Martínez, M. (2003). Una revisión sobre las concepciones alternativas de los estudiantes en relación con el equilibrio químico. Clasificación y síntesis de sugerencias didácticas. Educación Química 14(3), 60 - 66. DOI: http://dx.doi.org/10.22201/fq.18708404e.2003.3.66244 
Domínguez Prada, E.D., Cabrera Castillo, H. G. (2020). Análisis de objetos virtuales de aprendizaje centrados en el Principio de Le Chatelier.

Revista Convergencia Educativa, 7, julio, 1-21. DOI: http://doi.org/10.29035/rce.7.5

Roca, M., Márquez, C., \& Sanmartí, N. (2013). Las preguntas de los alumnos: una propuesta de análisis. Enseñanza de las Ciencias, 31(1), 95-114. Recuperado de https://www.raco.cat/index.php/Ensenanza/article/view/285706

Rodas, J. (2012). Estudio para determinar el uso y aplicación de las TIC: en los procesos de enseñanza aprendizaje por parte de los docentes de la ciudad de Milagro y cantones aledaños. UNEMI, 5(8), 79-92. DOI: https://doi.org/10.29076/issn.2528-7737vol5iss8.2012pp79-92p

Rojas, C. (2015). Objetos virtuales de aprendizaje como herramientas para la enseñanza del álgebra en el grado octavo de la Institución Educativa Ana de Castrillón (Tesis de Maestría). Universidad Nacional de Colombia, Medellín, Colombia. Recuperado de https://repositorio.unal.edu.co/handle/unal/55932

Ruiz, F. (2007). Modelos didácticos para la enseñanza de las ciencias naturales. Revista Latinoamericana de Estudios Educativos (Colombia), 3(2), 41-60.

Silva, M., \& Chica, P. (2016). Diseño y desarrollo de un objeto virtual de aprendizaje para un curso de electrónica. INGE CUC, 12(1), 9-20. DOI: https://doi.org/10.17981/ingecuc.12.1.2016.01

Sotomayor, C. (2015). Libros de texto digitales: consideraciones educativas. Funcae Digital, 58, 1-6. Recuperado de http://www.fundacionfuncae.es/archivos/documentosarticulos/SOTOMAYOR\%20MARTINEZ.pdf

Tabares., V. (2013). Modelo por capas para evaluación de la calidad de objetos de aprendizaje en repositorios de objetos de aprendizaje (Tesis de maestría). Universidad Nacional de Colombia, Medellín, Colombia. DOI: http://dx.doi.org/10.24320/redie.2017.19.3.1128

Talanquer, V. (2014). Desarrollando pensamiento químico en contextos sociales y ambientales. Educacio química EduQ, 17, 4-11.

Toll, Y., Ruiz, L., Trujillo, Y., \& Ril, Y. (2011). Calidad de los objetos de aprendizaje producidos en la universidad de las ciencias informáticas. Edutec-e. Revista Electrónica De Tecnología Educativa, 36, 1-18. Recuperado de https://www.edutec.es/revista/index.php/edutec-e/article/view/401/137 
Domínguez Prada, E.D., Cabrera Castillo, H. G. (2020). Análisis de objetos virtuales de aprendizaje centrados en el Principio de Le Chatelier.

Revista Convergencia Educativa, 7, julio, 1-21. DOI: http://doi.org/10.29035/rce.7.5

\section{Datos de correspondencia}

Edgar Ricardo Domínguez Prada

Licenciado en educación básica con énfasis en ciencias naturales y educación ambiental.

Universidad del Valle, Colombia.

Email: edgar.dominguez@correounivalle.edu.co

Henry Giovany Cabrera Castillo

Doctor en Educación

Grupo de investigación: Ciencia, Educación y

Diversidad

Universidad del Valle, Colombia.

Email: henry.g.cabrera.correounivalle.edu.co

(c) (1) (2) Esta obra está bajo una Licencia de Creative Commons Reconocimiento-Nocomercial(C) Compartirlgual 4.0 Internacional. 